\title{
DENSE SUBSEMIGROUPS OF GENERALISED TRANSFORMATION SEMIGROUPS
}

\author{
AMORN WASANAWICHIT and YUPAPORN KEMPRASIT
}

(Received 16 November 2000; revised 11 October 2001)

\author{
Communicated by D. Easdown
}

\begin{abstract}
In 1986, Higgins proved that $T(X)$, the semigroup (under composition) of all total transformations of a set $X$, has a proper dense subsemigroup if and only if $X$ is infinite, and he obtained similar results for partial and partial one-to-one transformations. We consider the generalised transformation semigroup $T(X, Y)$ consisting of all total transformations from $X$ into $Y$ under the operation $\alpha * \beta=\alpha \theta \beta$, where $\theta$ is any fixed element of $T(Y, X)$. We show that this semigroup has a proper dense subsemigroup if and only if $X$ and $Y$ are infinite and $|Y \theta|=\min \{|X|,|Y|\}$, and we obtain similar results for partial and partial one-to-one transformations. The results of Higgins then become special cases.
\end{abstract}

2000 Mathematics subject classification: primary $20 \mathrm{M} 20$.

\section{Introduction and preliminaries}

If $U$ is a subsemigroup of a semigroup $S$, we say $d \in S$ is dominated by $U$ (or $U$ dominates $d$ ) if for any semigroup $T$ and for any homomorphisms $\varphi, \psi: S \rightarrow$ $T, \varphi|U=\psi| U$ implies $d \varphi=d \psi$. The set of all elements of $S$ dominated by $U$ is called the dominion of $U$ in $S$ and is denoted by $\operatorname{Dom}(U, S)$. Clearly, $U \subseteq$ $\operatorname{Dom}(U, S) \subseteq S$, and we say $U$ is dense in $S$ if $\operatorname{Dom}(U, S)=S$, in which case the inclusion map id $\operatorname{id}_{U} U \rightarrow S$ is 'epi' in the sense that if $\alpha, \beta: S \rightarrow T$ are homomorphisms and $\alpha|U=\beta| U$ then $\alpha=\beta$.

Quite surprisingly, there is a useful characterisation-namely, Isbell's Zigzag Theorem (see below) - of the elements of $\operatorname{Dom}(U, S)$ which has applications concerning epimorphisms and amalgams of semigroups, an exposition of which can be found in [4, Chapter 4].

(C) 2002 Australian Mathematical Society $1446-7887 / 2000 \$ A 2.00+0.00$ 
Little seems to be known about the existence of dense subsemigroups. In [7] Isbell constructed a finite semigroup having a proper dense subsemigroup, and in [2] Hall produced an example of a finite dense subsemigroup of an infinite semigroup. On the other hand, it is easy to show that no left [right] zero semigroup can have a proper dense subsemigroup, and the same can be proved for finite groups (see Theorem 1 below). If $X$ is a set, Higgins [3] showed that $T(X)$ has a proper dense subsemigroup if and only if $X$ is infinite, and that the same is true for the semigroup $P(X)$ of all partial transformations of $X$ and also for the symmetric inverse semigroup $I(X)$. In Section 2, we generalise Higgins' result by employing more direct and elementary arguments than in [3].

In $[8,9]$ Magill generalised the notion of a transformation semigroup as follows. Let $X$ and $Y$ be non-empty sets and let $T(X, Y)$ denote the set of all total transformations from $X$ into $Y$. Fix $\theta \in T(Y, X)$ and define an operation $*$ on $T(X, Y)$ by

$$
\alpha * \beta=\alpha \circ \theta \circ \beta
$$

for all $\alpha, \beta \in T(X, Y)$. Under this operation, $T(X, Y)$ is a semigroup which we denote by $(T(X, Y), \theta)$. Some of its properties were studied in $[10,12]$.

In [11] Sullivan took this one step further by considering the set $P(X, Y)$ of all partial transformations from $X$ into $Y$ (that is, all $\alpha: A \rightarrow B$ where $A \subseteq X, B \subseteq Y$ ). Then $(P(X, Y), \theta)$ is a semigroup under the above operation for any $\theta \in P(Y, X)$. In the same way, we can obtain a semigroup $(I(X, Y), \theta)$ where $I(X, Y)$ is the set of all one-to-one partial transformations from $X$ into $Y$ and $\theta \in I(Y, X)$.

Throughout this paper, $(S(X, Y), \theta)$, or more briefly $(S, \theta)$, will denote one of the three transformation semigroups on $X, Y$ just introduced. Also, for any $\alpha \in P(X, Y)$, we will let $r(\alpha)=|X \alpha|$ and call this the rank of $\alpha$ (other notation and terminology will come from [1]). Our aim in Section 2 is to prove the following result.

THEOREM. For any sets $X$ and $Y$, if $S(X, Y)$ denotes $T(X, Y), P(X, Y)$ or $I(X, Y)$ and $\theta \in S(Y, X)$ then the semigroup $(S(X, Y), \theta)$ has a proper dense subsemigroup if and only if $X$ and $Y$ are both infinite and $r(\theta)=\min \{|X|,|Y|\}$.

Moreover, in proving this result, we show that under the given conditions $(S(X, Y), \theta)$ contains infinitely many proper dense subsemigroups.

We let $G(X)$ denote the symmetric group on a set $X$ and, if $A \subseteq X$, we let id $_{A}$ denote the identity mapping on $A$. Then, in particular, $P(X)=\left(P(X, X), \mathrm{id}_{X}\right)$, $I(X)=\left(I(X, X), \mathrm{id}_{X}\right)$ and $T(X)=\left(T(X, X), \mathrm{id}_{X}\right)$.

From the above theorem, we immediately deduce the following result.

COROLLARY (Higgins' Theorem [3]). If $X$ is a set and $S$ denotes any one of $T(X)$, $P(X)$ or $I(X)$ then $S$ has a proper dense subsemigroup if and only if $X$ is infinite. 
When $U$ is a subsemigroup of a semigroup $S$, a useful criterion for membership of $\operatorname{Dom}(U, S)$ is provided by Isbell's Zigzag Theorem [6]. A zigzag in $S$ over $U$ with value $d \in S$ is a system of equalities

$$
\begin{gathered}
d=u_{0} y_{1}, \quad u_{0}=x_{1} u_{1}, \\
u_{2 i-1} y_{i}=u_{2 i} y_{i+1}, \quad x_{i} u_{2 i}=x_{i+1} u_{2 i+1} \quad(i=1, \ldots, m-1), \\
u_{2 m-1} y_{m}=u_{2 m}, \quad x_{m} u_{2 m}=d,
\end{gathered}
$$

where $u_{0}, u_{1}, \ldots, u_{2 m} \in U$ and $x_{1}, \ldots, x_{m}, y_{1}, \ldots, y_{m} \in S$. Note that, if $d \notin U$ then, by choosing $m$ to be minimal, we may assume the zigzag is such that $x_{1}, \ldots, x_{m} \notin U$ and $y_{1}, \ldots, y_{m} \notin U$.

THEOREM (Isbell's Zigzag Theorem). Let $U$ be a subsemigroup of a semigroup $S$. Then $d \in \operatorname{Dom}(U, S)$ if and only if $d \in U$ or there is a zigzag in $S$ over $U$ with value $d$.

As a corollary to the Zigzag Theorem, Howie and Isbell [5] proved the following.

THEOREM 1. If $U$ is a subgroup of a semigroup $S$ then $\operatorname{Dom}(U, S)=U$. Hence, if $U$ is a subsemigroup of a finite group $G$ then $\operatorname{Dom}(U, G)=U$.

We remark that in the proof of his result, Higgins treats each of the three semigroups separately, and his arguments depend on Isbell's Zigzag Theorem and Theorem 1, as well as a result of Vorobev [13] (also see [1, page 7]): namely, if $X$ is a finite set then $T(X)$ is generated by $G(X) \cup\{\alpha\}$ where $\alpha$ is any element of $T(X)$ with rank $|X|-1$, and also a result of Hall [2]: namely, if $U$ is a proper regular subsemigroup of a finite semigroup $S$ then $\operatorname{Dom}(U, S) \neq S$. In our arguments below, we also employ the Zigzag Theorem and Theorem 1, but we avoid the results of Vorobev and Hall.

\section{A generalisation of Higgins' Theorem}

In this section, we use elementary concepts of mappings and cardinals to prove some lemmas concerning generalised transformation semigroups: they will culminate in a proof of our main theorem.

LEMMA 2. Let $\theta \in S(Y, X)$ be such that $r(\theta)<\min \{|X|,|Y|\}$.

(i) If $X$ or $Y$ is finite then, for every $\alpha \in S(X, Y), \operatorname{ran} \theta \alpha=\operatorname{ran} \alpha$ implies that there exists $\beta \in S(X, Y)$ such that $r(\beta)>r(\alpha)$ and $\theta \beta=\theta \alpha$.

(ii) If $X$ and $Y$ are infinite then, for every $\alpha \in S(X, Y)$, there exists $\beta \in S(X, Y)$ such that $r(\beta)=\min \{|X|,|Y|\}$ and $\theta \beta=\theta \alpha$. 
Proof. (i) Suppose $X$ or $Y$ is finite. By assumption, we have:

$$
r(\alpha)=r(\theta \alpha) \leq r(\theta)<\min \{|X|,|Y|\}<\aleph_{0} .
$$

Hence, $\operatorname{ran} \theta \subset X$ and $\operatorname{ran} \alpha \subset Y$, and both $\operatorname{ran} \theta$ and $\operatorname{ran} \alpha$ are finite. Let $a \in X \backslash \operatorname{ran} \theta$, $b \in Y \backslash \operatorname{ran} \alpha$ and define $\beta: \operatorname{dom} \alpha \cup\{a\} \rightarrow Y$ by:

$$
x \beta= \begin{cases}b & \text { if } x=a ; \\ x \alpha & \text { if } x \in \operatorname{dom} \alpha \backslash\{a\} .\end{cases}
$$

Clearly, $\beta \in S(X, Y)$ for the case when $S(X, Y)$ equals $T(X, Y)$ or $P(X, Y)$. And it is also true when $S(X, Y)=I(X, Y)$ since $b \notin \operatorname{ran} \alpha$. Also, $\theta \beta=\theta \alpha$ since $a \notin \operatorname{ran} \theta$.

We claim that $\operatorname{ran} \beta=\operatorname{ran} \alpha \cup\{b\}$, which implies that $r(\beta)>r(\alpha)$ since $\operatorname{ran} \alpha$ is finite. By definition of $\beta, \operatorname{ran} \beta=(\operatorname{dom} \alpha \backslash\{a\}) \alpha \cup\{b\}$. Hence, if $a \notin \operatorname{dom} \alpha$, the claim is valid. On the other hand, if $a \in \operatorname{dom} \alpha$, then $a \alpha \in \operatorname{ran} \alpha=\operatorname{ran} \theta \alpha$, so $a \alpha=z \alpha$ for some $z \in \operatorname{ran} \theta \cap \operatorname{dom} \alpha$, and hence

$$
\operatorname{ran} \alpha=(\operatorname{dom} \alpha \backslash\{a\}) \alpha \cup\{a \alpha\}=((\operatorname{dom} \alpha \backslash\{a\}) \cup\{z\}) \alpha=(\operatorname{dom} \alpha \backslash\{a\}) \alpha,
$$

thus the claim is valid in this case also.

(ii) Suppose $X$ and $Y$ are infinite. By assumption $r(\theta)<|X|$ and $r(\theta)<|Y|$. Hence, since $r(\theta \alpha) \leq r(\theta)$ and $X, Y$ are infinite, we have $|X \backslash \operatorname{ran} \theta|=|X|$ and $|Y \backslash \operatorname{ran} \theta \alpha|=|Y|$.

Case 1. $|X| \leq|Y|$. Let $\gamma$ be any one-to-one map from $X \backslash \operatorname{ran} \theta$ into $Y \backslash \operatorname{ran} \theta \alpha$, and define $\beta \in P(X, Y)$ by

$$
x \beta= \begin{cases}x \alpha & \text { if } x \in \operatorname{ran} \theta \cap \operatorname{dom} \alpha ; \\ x \gamma & \text { if } x \in X \backslash \operatorname{ran} \theta .\end{cases}
$$

Note that if $S(X, Y)=T(X, Y)$ then $\operatorname{dom} \alpha=X$ and so $\beta \in T(X, Y)$. Likewise, if $\alpha$ is one-to-one then so is $\beta$. Also, since $r(\theta \alpha)<|X|, r(\beta)=|X|=\min \{|X|,|Y|\}$. In addition, we have $\operatorname{dom} \theta \beta=\operatorname{dom} \theta \alpha$, and it follows that $\theta \beta=\theta \alpha$.

Case 2. $|X|>|Y|$. This implies $|X \backslash \operatorname{ran} \theta|>|Y \backslash \operatorname{ran} \theta \alpha|$, so we can choose $A \subseteq X \backslash \operatorname{ran} \theta$ with the same cardinal as $Y \backslash \operatorname{ran} \theta \alpha$ and let $\gamma$ be any bijection from $A$ onto $Y \backslash \operatorname{ran} \theta \alpha$. Define $\beta \in P(X, Y)$ by

$$
x \beta= \begin{cases}x \alpha & \text { if } x \in(X \backslash A) \cap \operatorname{dom} \alpha ; \\ x \gamma & \text { if } x \in A,\end{cases}
$$

and note that, as before, if $S(X, Y)=T(X, Y)$ then $\beta \in T(X, Y)$. Also, since $\operatorname{ran} \beta \subseteq Y, r(\beta)=|A|=|Y|=\min \{|X|,|Y|\}$. In addition, since $\operatorname{ran} \theta \subseteq X \backslash A$, we have $\operatorname{dom} \theta \beta=\operatorname{dom} \theta \alpha$, and it follows that $\theta \beta=\theta \alpha$. 
That completes the proof in this case if $S(X, Y)$ equals $P(X, Y)$ or $T(X, Y)$. If $S(X, Y)=I(X, Y)$, we choose $A$ and $\gamma$ as before, and define $\beta^{\prime} \in P(X, Y)$ by

$$
x \beta^{\prime}= \begin{cases}x \alpha & \text { if } x \in \operatorname{ran} \theta \cap \operatorname{dom} \alpha ; \\ x \gamma & \text { if } x \in A .\end{cases}
$$

Then, since $\operatorname{ran} \theta \subseteq X \backslash A$ and $\alpha$ is one-to-one, $\beta^{\prime}$ is also. And an argument similar to the one before shows that $\beta^{\prime}$ is the required mapping.

The next result shows that, under certain conditions, $S(X, Y)$ contains infinitely many proper subsemigroups. For convenience, we write $\left\{x_{i}\right\}$ to denote $\left\{x_{i}: i \in I\right\}$ where the index set $I$ can be deduced from context.

LEMMA 3. Let $X$ and $Y$ be infinite sets. Suppose $\theta \in S(Y, X)$ has infinite rank and choose an infinite subset $A$ of $\operatorname{ran} \theta$ such that $|\operatorname{ran} \theta \backslash A|=r(\theta)$. For each $a \in A$, choose $y_{a} \in a \theta^{-1}$ and let $U=\left\{\alpha \in S(X, Y):\left|A \alpha \cap\left(Y \backslash\left\{y_{a}\right\}\right)\right|<|A|\right\}$. Then $U$ is a proper subsemigroup of $(S(X, Y), \theta)$.

ProOF. Clearly, $U$ contains every $\alpha \in S(X, Y)$ with finite rank. To show $U$ is closed under the operation $*$, we let $\left\{y_{a}\right\}^{c}$ denote $Y \backslash\left\{y_{a}\right\}$ and observe that if $\alpha, \beta \in U$ then

$$
\begin{aligned}
A(\alpha \theta \beta) \cap\left\{y_{a}\right\}^{c} & =\left[\left(A \alpha \cap\left\{y_{a}\right\}^{c}\right) \theta \beta \cup\left(A \alpha \cap\left\{y_{a}\right\}\right) \theta \beta\right] \cap\left\{y_{a}\right\}^{c} \\
& \subseteq\left[\left(A \alpha \cap\left\{y_{a}\right\}^{c}\right) \theta \beta \cup\left(\left\{y_{a}\right\}\right) \theta \beta\right] \cap\left\{y_{a}\right\}^{c} \\
& \subseteq\left(A \alpha \cap\left\{y_{a}\right\}^{c}\right) \theta \beta \cup\left(A \beta \cap\left\{y_{a}\right\}^{c}\right)
\end{aligned}
$$

and hence $\alpha \theta \beta \in U$. To show $U$ is a proper subset of $S(X, Y)$, first note that $\left\{y_{a}\right\} \subseteq A \theta^{-1}$, so $\operatorname{dom} \theta \backslash A \theta^{-1} \subseteq \operatorname{dom} \theta \backslash\left\{y_{a}\right\} \subseteq Y \backslash\left\{y_{a}\right\}$. Therefore,

$$
|\operatorname{ran} \theta \backslash A| \leq\left|\operatorname{dom} \theta \backslash A \theta^{-1}\right| \leq\left|Y \backslash\left\{y_{a}\right\}\right| .
$$

Since $|A| \leq|\operatorname{ran} \theta|=|\operatorname{ran} \theta \backslash A|$, we can therefore choose a one-to-one mapping $\mu$ from $A$ into $Y \backslash\left\{y_{a}\right\}$. Then $\left|A \mu \cap\left(Y \backslash\left\{y_{a}\right\}\right)\right|=|A \mu|=|A|$, so $\mu \in S(X, Y) \backslash U$ if $S(X, Y)$ equals $P(X, Y)$ or $I(X, Y)$. If $S(X, Y)=T(X, Y)$, we let $\mu^{\prime}$ be any extension of $\mu$ to the whole of $X$ : that is, $A \mu^{\prime}=A \mu$ and so $\mu^{\prime} \in T(X, Y) \backslash U$.

Recall that each $\alpha \in S(X, Y)$ induces an equivalence $\alpha \circ \alpha^{-1}$ on its domain in $X$. The next result bears comparison with the characterisation of Green's $\mathscr{L}$ and $\mathscr{R}$ relations on $T(X)([1$, Lemma 2.5 and Lemma 2.6]). Its proof is routine and therefore is omitted.

LEMMA 4. The following statements hold for $S(X, Y)$ and any sets $X, Y$. 
(i) If $\alpha \in S(X, Y), \beta \in S(X, X)$ and $\alpha \circ \alpha^{-1}=\beta \circ \beta^{-1}$, then there exists $\mu \in S(X, Y)$ such that $\alpha=\beta \mu$.

(ii) If $\alpha \in S(X, Y), \beta \in S(Y, Y)$ and $\operatorname{ran} \alpha \subseteq \operatorname{ran} \beta$, then there exists $\mu \in S(X, Y)$ such that $\alpha=\mu \beta$.

Lemma 5. Let $\theta \in S(Y, X)$ and let $U$ be a dense subsemigroup of $(S(X, Y), \theta)$. Then $\{\alpha \in S(X, Y): r(\alpha)>r(\theta)\} \subseteq U$.

PROOF. By the Zigzag Theorem, if $\alpha \in S(X, Y) \backslash U$, then $\alpha=\beta \theta \gamma$ for some $\beta \in U, \gamma \in S(X, Y)$ and this implies $r(\alpha) \leq r(\theta)$.

LEMMA 6. Let $\theta \in S(Y, X)$ and let $U$ be a dense subsemigroup of $(S(X, Y), \theta)$ and $r(\theta)<\min \{|X|,|Y|\}$. Then $U=S(X, Y)$.

Proof. Write $S=S(X, Y)$ and suppose $S \backslash U \neq \emptyset$.

Case 1. $X$ or $Y$ is finite. Note that for all $\alpha \in S, r(\alpha) \leq \min \{|X|,|Y|\}<\aleph_{0}$. Hence, there exists $\beta \in S \backslash U$ with maximal rank. Since $U$ is dense in $(S, \theta)$, the Zigzag Theorem implies that $\beta=\lambda \theta \gamma$ for some $\lambda \in U, \gamma \in S \backslash U$. Then, using the maximality of $r(\beta)$, we have $r(\beta) \leq r(\theta \gamma) \leq r(\gamma) \leq r(\beta)$ and equality follows. But $\operatorname{ran} \theta \gamma \subseteq \operatorname{ran} \gamma$, and these are two sets of the same finite size, so they are equal. Hence, by Lemma 2 (i), there exists $\mu \in S$ such that $r(\mu)>r(\gamma)$ and $\theta \mu=\theta \gamma$. Then $r(\mu)>r(\beta)$ and, by choice of $\beta$, this means $\mu \in U$. So, we have $\beta=\lambda \theta \gamma=\lambda \theta \mu=\lambda * \mu \in U$, a contradiction.

Case 2. $X$ and $Y$ are infinite. By the Zigzag Theorem, if $\eta \in S \backslash U$, then $\eta=\lambda \theta \gamma$ for some $\lambda \in U$ and $\gamma \in S$. By Lemma 2 (ii), $\theta \gamma=\theta \beta$ for some $\beta \in S$ with $r(\beta)=\min \{|X|,|Y|\}$. This and the supposition imply that $r(\beta)>r(\theta)$ and so $\beta \in U$ by Lemma 5 . Hence, $\eta=\lambda \theta \gamma=\lambda \theta \beta \in U$, a contradiction.

The next two results will be used to show that if $X$ or $Y$ is finite then $S(X, Y)$ cannot have a proper dense subsemigroup. In the proof of the first we rely on the simple observation that if $U$ is a dense subsemigroup of a semigroup $S$ and $\rho$ is a congruence on $S$ then $\{x \rho: x \in U\}$ is a dense subsemigroup of the semigroup $S / \rho$.

LEMMA 7. Let $X, Y$ be arbitrary sets with $X$ finite. Suppose $\theta \in S(Y, X), \operatorname{ran} \theta=$ $X$ and $U$ is a dense subsemigroup of $(S(X, Y), \theta)$. Then $U=S(X, Y)$.

ProOF. Write $S=S(X, Y)$. Since $\operatorname{ran} \theta=X$, there exists a one-to-one mapping $\gamma: X \rightarrow Y$ such that $\gamma \theta=\mathrm{id}_{X}$. Then $\gamma \in S$. Next we let $V=\{\alpha \in S: \alpha \theta \in G(X)\}$ and define a relation $\rho$ on $V$ (compare Symons' $\ell$-relation in [11]) by

$$
(\alpha, \beta) \in \rho \quad \text { if and only if } \alpha \theta=\beta \theta .
$$

To show $U=S$, consider the following statements. 
(1) $V$ is a subsemigroup of $(S, \theta)$.

(2) For $\alpha \in S, \operatorname{ran} \alpha \theta=X$ implies that $\alpha \in V$.

(3) For $\alpha, \beta \in S, \alpha \theta \beta \in V$ implies that $\alpha, \beta \in V$.

(4) $\rho$ is a congruence on $V$ and $V / \rho$ is isomorphic to $G(X)$.

(5) If $V \nsubseteq U$ then $U \cap V$ is a dense subsemigroup of $V$.

(6) $V \subseteq U$.

The proofs of (1), (2), (3) and the first half of (4) are straightforward. The second half of (4) follows from the fact that the mapping: $V \rightarrow G(X), \alpha \mapsto \alpha \theta$ is an epimorphism whose kernel is $\rho$.

Let $\eta \in V \backslash U$. Since $U$ is dense in $S$, the Zigzag Theorem implies there is a zigzag, $Z$ say, in $S$ over $U$ with value $\eta$. Since $\eta \in V$, it follows from (3) that $Z$ is a zigzag in $V$ over $U \cap V$ with value $\eta$. This proves $U \cap V$ is a dense subsemigroup of $V$, so (5) is proved.

Suppose (6) does not hold and let $\eta \in V \backslash U$. By (5), $\{\alpha \rho: \alpha \in U \cap V\}$ is a dense subsemigroup of $V / \rho$. Since $G(X)$ is a finite group, Theorem 1 implies $G(X)$ has no proper dense subsemigroup, and so $\{\alpha \rho: \alpha \in U \cap V\}=V / \rho$. Hence, from the definition of $\rho$, for each $\alpha \in V$ there exists $\alpha^{\prime} \in U \cap V$ such that $\alpha \theta=\alpha^{\prime} \theta$. Since $U \cap V$ is dense in $V$ and $\eta \in V \backslash U$, the Zigzag Theorem implies $\eta=\alpha \theta \lambda$ for some $\alpha \in V, \lambda \in U \cap V$. Then $\eta=\alpha^{\prime} \theta \lambda=\alpha^{\prime} * \lambda \in U \cap V$, a contradiction. Thus (6) holds.

Now we prove $U=S$. Suppose $U \neq S$ and note that $r(\alpha \theta) \leq|X|<\aleph_{0}$ for all $\alpha \in S$. Hence there exists $\mu \in S \backslash U$ such that $r(\mu \theta)$ is maximal. Since $\mu \notin U$ and $V \subseteq U$ by (6), we deduce from (2) that $\operatorname{ran} \mu \theta \subset X$, and so $r(\mu \theta)<|X|$ since $X$ is finite. In addition, the Zigzag Theorem implies $\mu=\lambda \theta \beta$ for some $\lambda \in U$ and $\beta \in S \backslash U$. Hence, using the maximality of $r(\mu \theta)$, we have:

$$
r(\mu \theta) \leq r(\beta \theta) \leq r(\mu \theta),
$$

and equality follows. Let $x_{0} \in X \backslash \operatorname{ran} \beta \theta$ and $x_{1} \in X \backslash \operatorname{ran} \lambda \theta$. Since $\operatorname{ran} \theta=X$ (by assumption), we can choose $y \in Y$ such that $y \theta=x_{0}$ and define $\beta^{\prime}: \operatorname{dom} \beta \cup\left\{x_{1}\right\} \rightarrow Y$ by

$$
x \beta^{\prime}= \begin{cases}x \beta & \text { if } x \in \operatorname{dom} \beta \backslash\left\{x_{1}\right\} \\ y & \text { if } x=x_{1} .\end{cases}
$$

Clearly, $\beta^{\prime} \in S$ if $S$ equals $T(X, Y)$ or $P(X, Y)$. If $y=x \beta^{\prime}$ for some $x \in \operatorname{dom} \beta \backslash\left\{x_{1}\right\}$, then $x_{0}=y \theta=x \beta^{\prime} \theta=x \beta \theta \in \operatorname{ran} \beta \theta$, contradicting the choice of $x_{0}$. Hence, $\beta^{\prime} \in S$ if $S=I(X, Y)$.

Now, since $\beta$ and $\beta^{\prime}$ agree on $\operatorname{dom} \beta \backslash\left\{x_{1}\right\}$ and $x_{1} \notin \operatorname{ran} \lambda \theta$, we have $\lambda \theta \beta^{\prime}=\lambda \theta \beta=$ $\mu$. Clearly, $\operatorname{ran} \mu \theta \subseteq \operatorname{ran} \beta \theta$ and, as already shown, these two sets have the same 
finite size, hence they are equal. Therefore,

$$
\begin{aligned}
r\left(\beta^{\prime} \theta\right)=\left|X \beta^{\prime} \theta\right| & \geq\left|\left(\operatorname{ran} \lambda \theta \cup\left\{x_{1}\right\}\right) \beta^{\prime} \theta\right| \\
& =\left|\left(\operatorname{ran} \lambda \theta \beta^{\prime}\right) \theta \cup\left\{x_{0}\right\}\right|=\left|\operatorname{ran} \mu \theta \cup\left\{x_{0}\right\}\right| \\
& =r(\beta \theta)+1>r(\mu \theta) .
\end{aligned}
$$

It follows from the maximality of $r(\mu \theta)$ that $\beta^{\prime} \in U$. Hence, $\mu=\lambda \theta \beta^{\prime}=\lambda * \beta^{\prime} \in U$, a contradiction. Therefore, $U=S$ as required.

LEMMA 8. Let $X, Y$ be arbitrary sets with $Y$ finite. Suppose $\theta \in S(Y, X), \operatorname{dom} \theta=$ $Y$ and $U$ is a dense subsemigroup of $(S(X, Y), \theta)$. Then $U=S(X, Y)$.

Proof. Write $S=S(X, Y)$. We have $r(\theta) \leq|\operatorname{dom} \theta|=|Y|<\aleph_{0}$. Also, either $r(\theta)=|X|$ or $r(\theta)=|Y|$ or $r(\theta)<\min \{|X|,|Y|\}$. If the first occurs, then $X$ is finite and $\operatorname{ran} \theta=X$, so Lemma 7 implies $U=S$. If the last occurs, then $U=S$ by Lemma 6.

Hence we assume $r(\theta)=|Y|$. In this event, the domain and range of $\theta$ have the same finite size, $\theta$ is one-to-one and $|Y| \leq|X|$. Let $V=\{\alpha \in S:(\operatorname{ran} \theta) \alpha=Y\}$ and define a relation $\rho$ on $V$ by $(\alpha, \beta) \in \rho$ if and only if $\theta \alpha=\theta \beta$. To show $U=S$, consider the following statements.

(1) $V$ is a subsemigroup of $(S, \theta)$.

(2) For $\alpha, \beta \in S$, $\operatorname{ran} \alpha \theta \beta=Y$ implies that $\beta \in V$.

(3) For $\alpha, \beta \in S, \alpha \theta \beta \in V$ implies that $\alpha, \beta \in V$.

(4) $\rho$ is a congruence on $V$ and $V / \rho$ is isomorphic to $G(Y)$.

(5) If $V \nsubseteq U$ then $U \cap V$ is a dense subsemigroup of $V$.

(6) $V \subseteq U$.

(7) For $\alpha \in S, \operatorname{ran} \alpha=Y$ implies that $\alpha \in U$.

The proofs of (1), (2), (3) and the first half of (4) are straightforward.

Since $r(\theta)=|Y|$, we know that $G(\operatorname{ran} \theta)$ is isomorphic to $G(Y)$ so, to complete the proof of (4), it suffices to prove that $V / \rho$ is isomorphic to $G(\operatorname{ran} \theta)$. But this follows immediately since the map: $V \rightarrow G(\operatorname{ran} \theta), \alpha \mapsto(\alpha \mid \operatorname{ran} \theta) \theta$ is clearly an epimorphism whose kernel is $\rho$.

Suppose there exists $\eta \in V \backslash U$ and let $Z$ be a zigzag in $S$ over $U$ with value $\eta$. It follows from (3) that $Z$ is a zigzag in $V$ over $U \cap V$ with value $\eta$. This proves that $U \cap V$ is a dense subsemigroup of $V$ which verifies (5).

Suppose (6) does not hold, so there exists $\eta \in V \backslash U$. By (5) and an observation before the statement of Lemma 7, $\{\alpha \rho: \alpha \in U \cap V\}$ is a dense subsemigroup of $V / \rho$. Since $Y$ is finite and $V / \rho$ is isomorphic to $G(Y)$, Theorem 1 implies that $V / \rho$ has no proper dense subsemigroup. Hence $\{\alpha \rho: \alpha \in U \cap V\}=V / \rho$. But, since $U \cap V$ is dense in $V$ and $\eta \in V \backslash U$, the Zigzag Theorem implies that $\eta=\lambda \theta \beta$ for 
some $\lambda \in U \cap V$ and $\beta \in V$. Then $\beta|\operatorname{ran} \theta=\mu| \operatorname{ran} \theta$ for some $\mu \in U \cap V$. Thus, $\theta \beta=\theta \mu$ and so $\eta=\lambda \theta \beta=\lambda \theta \mu=\lambda * \mu \in U \cap V$, contradicting the choice of $\eta$. Therefore, $V \subseteq U$ and thus (6) holds.

Let $\alpha \in S$ and $\operatorname{ran} \alpha=Y$, and suppose $\alpha \notin U$. Then by the Zigzag Theorem, $\alpha=\beta \theta \gamma$ for some $\beta \in U, \gamma \in S$. Hence, $Y=\operatorname{ran} \beta \theta \gamma$, and so $\gamma \in V \subseteq U$ by (2) and (6). Consequently, $\alpha=\beta * \gamma \in U$, a contradiction. Therefore, (7) holds.

Now we prove $U=S$. Suppose $U \neq S$. Since for all $\alpha \in S, r(\alpha) \leq|Y|<\aleph_{0}$, it follows that there exists $\mu \in S \backslash U$ with maximal rank. By (7) this means $r(\mu)<|Y|$. Since $U$ is dense in $(S, \theta)$ and $\mu \in S \backslash U$, the Zigzag Theorem implies $\mu=\lambda_{0} \theta \gamma=$ $\left(\beta \theta \lambda_{1}\right) \theta \gamma$ for some $\lambda_{0}, \lambda_{1} \in U$ and $\beta, \gamma \in S \backslash U$ such that $\lambda_{0}=\beta \theta \lambda_{1}$. Then, using the maximality of $r(\mu)$, we have:

$$
r(\mu) \leq r\left(\lambda_{0}\right) \leq r(\beta) \leq r(\mu), \quad r(\mu) \leq r(\gamma) \leq r(\mu),
$$

and equality follows throughout. Then $\operatorname{ran} \mu=\operatorname{ran} \gamma \operatorname{since} \operatorname{ran} \mu \subseteq \operatorname{ran} \gamma$ and these two sets have the same finite size.

We claim that there exists $\theta^{\prime} \in S(Y, X)$ such that $\lambda_{0} \theta^{\prime}=\lambda_{0} \theta, \operatorname{ran} \theta^{\prime} \gamma=\operatorname{ran} \gamma$ and $r\left(\theta^{\prime}\right)<|Y|$. To prove this, let $\theta_{0}: Y \rightarrow X$ be such that

$$
\theta_{0}\left|\operatorname{ran} \lambda_{0}=\theta\right| \operatorname{ran} \lambda_{0} \quad \text { and } \quad\left(Y \backslash \operatorname{ran} \lambda_{0}\right) \theta_{0} \subseteq\left(\operatorname{ran} \lambda_{0}\right) \theta .
$$

Then $\theta_{0} \in T(Y, X)$ and $\operatorname{ran} \theta_{0}=\left(\operatorname{ran} \lambda_{0}\right) \theta$. Put $\theta^{\prime}=\theta_{0}$ if $S=T(X, Y)$ and $\theta^{\prime}=\theta \mid \operatorname{ran} \lambda_{0}$ in the other two cases. Then $\theta^{\prime} \in S(Y, X), \lambda_{0} \theta^{\prime}=\lambda_{0} \theta$ and $r\left(\theta^{\prime}\right)=$ $\left|\left(\operatorname{ran} \lambda_{0}\right) \theta\right| \leq r\left(\lambda_{0}\right)<|Y|$. Also,

$$
\operatorname{ran} \theta^{\prime} \gamma=\left(\operatorname{ran} \lambda_{0} \theta\right) \gamma=\operatorname{ran}\left(\lambda_{0} \theta \gamma\right)=\operatorname{ran} \mu=\operatorname{ran} \gamma,
$$

and the claim is valid.

Now we have $r\left(\theta^{\prime}\right)<\min \{|X|,|Y|\}$ (since $|Y| \leq|X|$, as we observed at the start) and $\operatorname{ran} \theta^{\prime} \gamma=\operatorname{ran} \gamma$. Then by Lemma 2 (i), there exists $\eta \in S$ such that $r(\eta)>r(\gamma)$ and $\theta^{\prime} \eta=\theta^{\prime} \gamma$. Therefore, $r(\eta)>r(\mu)$, so $\eta \in U$ by choice of $\mu$. Moreover, since $\lambda_{0} \theta=\lambda_{0} \theta^{\prime}$ and $\theta^{\prime} \gamma=\theta^{\prime} \eta$, we have

$$
\mu=\left(\lambda_{0} \theta\right) \gamma=\lambda_{0} \theta^{\prime} \gamma=\lambda_{0}\left(\theta^{\prime} \eta\right)=\lambda_{0} \theta \eta .
$$

Consequently, $\mu=\lambda_{0} * \eta \in U$, contradicting the choice of $\mu$, and the proof is complete.

The next result enables us to construct proper dense subsemigroups of $(S(X, Y), \theta)$.

LEMMA 9. Let $X, Y$ be infinite sets. Suppose $\theta \in S(Y, X)$ and $r(\theta)=\min \{|X|,|Y|\}$. Let $A$ be an infinite subset of $\operatorname{ran} \theta$ such that $|\operatorname{ran} \theta \backslash A|=r(\theta)$ and, for each $a \in A$, choose $z_{a} \in a \theta^{-1}$. Put $U=\left\{\alpha \in S(X, Y):\left|A \alpha \cap\left(Y \backslash\left\{z_{a}\right\}\right)\right|<|A|\right\}$. Then $U$ is a proper dense subsemigroup of $(S(X, Y), \theta)$. 
Proof. Write $S=S(X, Y)$. By Lemma $3, U$ is a proper subsemigroup of $(S, \theta)$. To show that $U$ is dense in $(S, \theta)$, we have to show $S \subseteq \operatorname{Dom}(U, S)$; and for this, we must prove that for each $\alpha \in S$ there is a zigzag in $S$ over $U$ with value $\alpha$. To do this, we first construct a one-to-one mapping:

$$
\varphi:\left\{y \alpha^{-1}: y \in \operatorname{ran} \alpha\right\} \rightarrow\left\{x \theta^{-1}: x \in \operatorname{ran} \theta\right\}
$$

as follows. For each $y \in \operatorname{ran} \alpha$, choose $a_{y} \in y \alpha^{-1} \cap A$ provided this set is non-empty, and put

$$
\left(y \alpha^{-1}\right) \varphi \begin{cases}=a_{y} \theta^{-1} & \text { if } y \alpha^{-1} \cap A \neq \emptyset \\ \in\left\{x \theta^{-1}: x \in \operatorname{ran} \theta \backslash A\right\} & \text { if } y \alpha^{-1} \cap A=\emptyset .\end{cases}
$$

Note that, since $A \subseteq \operatorname{ran} \theta$, each $a_{y} \theta^{-1}$ is non-empty, and the mapping:

$$
\left\{y \alpha^{-1}: y \alpha^{-1} \cap A \neq \emptyset\right\} \rightarrow\left\{a_{y} \theta^{-1}: y \alpha^{-1} \cap A \neq \emptyset\right\}, y \alpha^{-1} \mapsto a_{y} \theta^{-1}
$$

is a bijection. By assumption, we have $\min \{|X|,|Y|\}=|\operatorname{ran} \theta \backslash A|$, so

$$
\begin{aligned}
\mid\left\{y \alpha^{-1}: y \in \operatorname{ran} \alpha \text { and } y \alpha^{-1} \cap A=\emptyset\right\} \mid & \leq r(\alpha) \leq \min \{|X|,|Y|\} \\
& =\left|\left\{x \theta^{-1}: x \in \operatorname{ran} \theta \backslash A\right\}\right| .
\end{aligned}
$$

In other words, it is possible to define $\varphi$ as in (1) so that $\varphi$ is one-to-one.

Now, from the definition of $\varphi$, we see that

$$
\bigcup\left\{\left(y \alpha^{-1}\right) \varphi: y \in \operatorname{ran} \alpha\right\} \subseteq \operatorname{dom} \theta
$$

and if $y \alpha^{-1} \cap A \neq \emptyset$ then $z_{a_{y}} \in a_{y} \theta^{-1}=\left(y \alpha^{-1}\right) \varphi$. Let $\lambda: \operatorname{dom} \alpha \rightarrow \operatorname{dom} \theta$ be a mapping with the property:

$$
\left(y \alpha^{-1}\right) \lambda \begin{cases}\subseteq\left(y \alpha^{-1}\right) \varphi & \text { if } y \in \operatorname{ran} \alpha \\ =\left\{z_{a_{y}}\right\} & \text { if } y \alpha^{-1} \cap A \neq \emptyset .\end{cases}
$$

From the supposition, we know $r(\lambda) \leq \min \{|X|,|Y|\}=r(\theta)=|\operatorname{ran} \theta \backslash A|$, and hence there exists $\eta_{1} \in I(X, Y)$ such that $\operatorname{dom} \eta_{1} \subseteq \operatorname{ran} \theta \backslash A$ and $\operatorname{ran} \eta_{1}=\operatorname{ran} \lambda$. Let $\eta_{2}: X \rightarrow Y$ be an extension of $\eta_{1}$ such that $\left|A \eta_{2}\right|<|A|$, and put $\eta=\eta_{2}$ if $S=T(X, Y)$ and $\eta=\eta_{1}$ in the other two cases. Then $\eta \in S$.

To complete the proof, we require the following statements.

(a) $\lambda, \eta \in U$.

(b) $\alpha \circ \alpha^{-1}=(\lambda \theta) \circ(\lambda \theta)^{-1}$.

(c) There exists $\gamma \in S$ such that $\alpha=\lambda \theta \gamma$.

(d) $\eta \theta \gamma \in U$. 
(e) There exists $\beta \in S$ such that $\lambda=\beta \theta \eta$.

If these statements hold, we have the following zigzag in $S$ over $U$ with value $\alpha$ :

$$
\begin{aligned}
\alpha & =\lambda \theta \gamma, & & \lambda \in U, \gamma \in S \\
\lambda & =\beta \theta \eta, & & \eta \in U, \beta \in S \\
\beta \theta(\eta \theta \gamma) & =\alpha, & & \eta \theta \gamma \in U .
\end{aligned}
$$

Therefore, by the Zigzag Theorem, $\alpha \in \operatorname{Dom}(U, S)$ as required.

To show that (a)-(e) hold, we proceed as follows.

(a) From the definition of $\lambda,\left(y \alpha^{-1} \cap A\right) \lambda=\left\{z_{a_{y}}\right\}$ whenever $y \alpha^{-1} \cap A \neq \emptyset$. Therefore, since $\operatorname{dom} \lambda=\operatorname{dom} \alpha$, we have

$$
\begin{aligned}
A \lambda=(A \cap \operatorname{dom} \alpha) \lambda & =\left[\cup\left\{A \cap y \alpha^{-1}: y \in \operatorname{ran} \alpha\right\}\right] \lambda \\
& =\left\{z_{a_{y}}: y \alpha^{-1} \cap A \neq \emptyset\right\} \subseteq\left\{z_{a}: a \in A\right\} .
\end{aligned}
$$

Hence, $A \lambda \cap\left(Y \backslash\left\{z_{a}\right\}\right)=\emptyset$ and thus $\lambda \in U$. From the definition of $\eta,|A \eta|=\left|A \eta_{2}\right|<|A|$ if $S=T(X, Y)$, and $|A \eta|=\left|A \eta_{1}\right|=0$ in the other two cases: this implies that $\eta \in U$.

(b) Since $\operatorname{dom} \lambda=\operatorname{dom} \alpha$ and $\operatorname{ran} \lambda \subseteq \operatorname{dom} \theta$, we have $\operatorname{dom} \lambda \theta=\operatorname{dom} \alpha$. That $\alpha \circ \alpha^{-1}=(\lambda \theta) \circ(\lambda \theta)^{-1}$ now follows readily from (1) and (2).

(c) This follows directly from (b) and Lemma 4 (i).

(d) This follows from the definition of $U$ and the fact that $|A(\eta \theta \gamma)| \leq|A \eta|<|A|$.

(e) From the definitions of $\eta_{1}$ and $\eta$, we have:

$$
\operatorname{ran} \lambda=\operatorname{ran} \eta_{1}=\left(\operatorname{dom} \eta_{1}\right) \eta_{1} \subseteq(\operatorname{ran} \theta) \eta_{1} \subseteq(\operatorname{ran} \theta) \eta=\operatorname{ran} \theta \eta
$$

Therefore, from Lemma 4 (ii), there exists $\beta \in S$ such that $\beta(\theta \eta)=\lambda$, as required.

We now restate the theorem presented in Section 1 with more details, and use the foregoing lemmas to prove it.

THEOREM 10. Suppose $X, Y$ are arbitrary sets. Let $S=S(X, Y)$ denote any one of $T(X, Y), P(X, Y)$ or $I(X, Y)$ and let $\theta \in S(Y, X)$. Then the semigroup $(S, \theta)$ has a proper dense subsemigroup if and only if $X$ and $Y$ are both infinite and $r(\theta)=\min \{|X|,|Y|\}$. Moreover, when this occurs, the following statements are true.

(1) Suppose $A$ is an infinite subset of $\operatorname{ran} \theta$ such that $|\operatorname{ran} \theta \backslash A|=r(\theta)$, and for each $a \in A$, choose $y_{a} \in a \theta^{-1}$. Then the set $U$ defined by

$$
U=\left\{\alpha \in S:\left|A \alpha \cap\left(Y \backslash\left\{y_{a}: a \in A\right\}\right)\right|<|A|\right\}
$$

is a proper dense subsemigroup of $(S, \theta)$. 
(2) $(S, \theta)$ has infinitely many proper dense subsemigroups, and the cardinality of the collection of all such subsemigroups is at least $\min \{|X|,|Y|\}$.

Proof. Suppose $X$ is finite or $Y$ is finite or $r(\theta)<\min \{|X|,|Y|\}$. If the last of these occurs, then $S$ has no proper dense subsemigroup by Lemma 6. If $r(\theta)=|X|$ then $X$ is finite and $\operatorname{ran} \theta=X$, whence $S$ has no proper dense subsemigroup by Lemma 7. If $r(\theta)=|Y|$ then $Y$ is finite and $\operatorname{dom} \theta=Y$, so the desired result follows from Lemma 8 . The converse, and statement (1) of the theorem, follow directly from Lemma 9.

To prove statement (2), assume $X$ and $Y$ are infinite and $r(\theta)=\min \{|X|,|Y|\}$. Since $|\operatorname{ran} \theta \times \operatorname{ran} \theta|=r(\theta)$, there exists a partition $\left\{A_{x}: x \in \operatorname{ran} \theta\right\}$ of $\operatorname{ran} \theta$ such that $\left|A_{x}\right|=r(\theta)$ for all $x \in \operatorname{ran} \theta$. Then $\left|\operatorname{ran} \theta \backslash A_{x}\right|=r(\theta)$ for all $x \in \operatorname{ran} \theta$. For each $x \in \operatorname{ran} \theta$, choose $y_{x} \in x \theta^{-1}$ and let

$$
U_{x}=\left\{\alpha \in S:\left|A_{x} \alpha \cap\left(Y \backslash\left\{y_{a}: a \in A_{x}\right\}\right)\right|<\left|A_{x}\right|\right\} .
$$

By Lemma 9, each $U_{x}$ is a proper dense subsemigroup of $(S, \theta)$. Moreover, let $x, x^{\prime}$ be distinct elements of $\operatorname{ran} \theta$. Then $\left|A_{x} \cup A_{x^{\prime}}\right|=\left|A_{x}\right|=\left|\left\{y_{a}: a \in A_{x}\right\}\right|$. Hence, there exists $\alpha \in I(X, Y)$ with $\operatorname{dom} \alpha=A_{x} \cup A_{x^{\prime}}$ and $\operatorname{ran} \alpha=\left\{y_{a}: a \in A_{x}\right\}$. Choose $\beta \in S$ such that $\beta \mid \operatorname{dom} \alpha=\alpha$. Then $A_{x} \beta \subseteq\left\{y_{a}: a \in A_{x}\right\}$ and

$$
A_{x^{\prime}} \beta \subseteq\left\{y_{a}: a \in A_{x}\right\} \subseteq Y \backslash\left\{y_{a}: a \in A_{x^{\prime}}\right\} .
$$

Hence, the intersection of $A_{x} \beta$ and $Y \backslash\left\{y_{a}: a \in A_{x}\right\}$ is empty, whereas the intersection of $A_{x^{\prime}} \beta$ with $Y \backslash\left\{y_{a}: a \in A_{x^{\prime}}\right\}$ has the same cardinality as $A_{x^{\prime}}$ (since $\alpha$ is one-to-one). That is, $\beta \in U_{x}$ but $\beta \notin U_{x^{\prime}}$. This shows the sets $U_{x}, x \in \operatorname{ran} \theta$, are all distinct, thereby verifying (2).

\section{Acknowledgements}

This paper formed part of a doctoral dissertation written under the supervision of the second author. He greatly appreciates the help of his supervisor in this work; and both authors are grateful for the assistance of Bob Sullivan, University of Western Australia, in the preparation of this paper. They also thank the referee and Dr David Easdown, University of Sydney, Australia, for their many helpful comments.

\section{References}

[1] A. H. Clifford and G. B. Preston, The algebraic theory of semigroups, Math. Surveys 7, Vol. 1 (Amer. Math. Soc., Providence, RI, 1961). 
[2] T. E. Hall, 'Epimorphisms and dominions', Semigroup Forum 24 (1982), $271-283$.

[3] P. M. Higgins, 'Dense subsets of some common classes of semigroup', Semigroup Forum 34 (1986), 5-19.

[4] - Techniques of semigroup theory (Oxford University Press, Oxford, 1992).

[5] J. M. Howie and J. R. Isbell, 'Epimorphisms and dominions II', J. Algebra 6 (1967), 7-21.

[6] J. R. Isbell, 'Epimorphisms and dominions', in: Proc. Conf. Categorical Algebra (La Jolla. Calif., 1965) (ed. S. Eilenberg et al.) (Springer, New York, 1966) pp. 232-246.

[7] _ - 'Notes on semigroup dominions', Semigroup Forum 7 (1974), 364-368.

[8] K. D. Magill, Jr., 'Semigroup structures for families of functions, I. Some homomorphism theorems', J. Austral. Math. Soc. 7 (1967), 81-94.

[9] _ 'Semigroup structures for families of functions, II. Continuous functions', J. Austral. Math. Soc. 7 (1967), 95-107.

[10] K. D. Magill, Jr., P. R. Misra and U. B. Tewari, 'Symons' $d$-congruence on sandwich semigroups', Czechoslovak Math. J. 33 (1983), 221-236.

[11] R. P. Sullivan, 'Generalised partial transformation semigroups', J. Austral. Math. Soc. 19 (1975), $470-473$.

[12] J. S. V. Symons, 'On a generalization of the transformation semigroup', J. Austral. Math. Soc. 19 (1975), 47-61.

[13] N. N. Vorobev, 'On symmetric associative systems', Leningrad Gos. Ped. Inst. Ucen. Zap. 89 (1953), 161-166 (Russian).

\section{Department of Mathematics}

Chulalongkorn University

Bangkok 10330

Thailand 
